\title{
Supporting Information: Reevaluating the Stability and Prevalence of Conglomerates: Implications for Preferential Crystallization
}

\author{
A. Otero-de-la-Roza, ${ }^{\dagger}$ Jason E. Hein, ${ }^{*,+}$ and Erin R. Johnson*, \\ Department of Chemistry, University of British Columbia, Okanagan, 3247 University \\ Way, Kelowna, British Columbia, Canada V1V $1 \mathrm{~V} 7$, Department of Chemistry, University \\ of British Columbia, 2036 Main Mall Vancouver, BC Canada V6T 1Z1, and Department of \\ Chemistry, Dalhousie University, 6274 Coburg Road, Halifax, Nova Scotia, Canada B3H \\ $4 R 2$ \\ E-mail: jhein@chem.ubc.ca; erin.johnson@dal.ca
}

*To whom correspondence should be addressed

${ }^{\dagger}$ University of British Columbia, Okanagan

$\ddagger$ University of British Columbia

"Dalhousie University 
Table 1: Crystallographic data codes and predicted enantiomeric excess (e.e. in \%) for the chiral crystal pairs. Also shown are the computed unit-cell volumes $\left(V\right.$ in $\left.\AA^{3}\right)$, the total lattice energies $\left(E_{\text {latt }}\right)$ and the dispersion contributions $\left(E_{\text {disp }}\right)$, both in $\mathrm{kcal} / \mathrm{mol}$ per molecule, for the homochiral $(c)$ and heterochiral $(r)$ crystals.

\begin{tabular}{|c|c|c|c|c|c|c|c|c|}
\hline Homochiral & Heterochiral & e.e. & $V_{c}$ & $V_{r}$ & $E_{\text {latt }, c}$ & $E_{\text {latt }, r}$ & $E_{\text {disp }, c}$ & $E_{\text {disp }, r}$ \\
\hline AFIHOO & WUNBEN & 0.00 & 483.86 & 479.24 & 39.38 & 38.88 & 48.15 & 48.26 \\
\hline CAPMSU & CXPMSO & 0.00 & 201.53 & 200.67 & 32.34 & 32.04 & 22.77 & 23.16 \\
\hline CIDJIJ & DAVHUF & 0.00 & 186.19 & 180.99 & 37.92 & 37.83 & 19.07 & 20.14 \\
\hline CIQSAW & CIQSEA & 0.00 & 265.39 & 276.61 & 39.84 & 39.59 & 31.04 & 27.96 \\
\hline DFBPAC & FBPACR & 0.00 & 203.33 & 205.91 & 33.73 & 33.52 & 23.75 & 23.09 \\
\hline FIRMIC & FIBKOQ & 0.00 & 390.13 & 389.36 & 38.68 & 37.85 & 42.27 & 42.21 \\
\hline GLYALB & GLYDLA & 0.00 & 171.40 & 164.66 & 57.00 & 56.79 & 19.11 & 20.40 \\
\hline HIVKOO & HIVKII & 0.00 & 286.84 & 288.12 & 33.99 & 33.41 & 30.73 & 30.26 \\
\hline LASPRT & DLASPA & 0.00 & 133.62 & 132.64 & 49.47 & 49.10 & 18.89 & 19.15 \\
\hline LGLUAC & YUYMOU & 0.00 & 153.03 & 150.62 & 52.07 & 51.08 & 21.87 & 22.65 \\
\hline LIYCUR & HOXCOM & 0.00 & 386.78 & 382.21 & 30.41 & 29.92 & 33.72 & 34.68 \\
\hline MIHTEE & HERMEX & 0.00 & 160.74 & 163.41 & 24.90 & 24.84 & 20.93 & 20.33 \\
\hline OGOHAT & OGOGUM & 0.00 & 215.70 & 222.32 & 28.81 & 28.53 & 26.38 & 24.50 \\
\hline OPADNA & OPADNB & 0.00 & 228.48 & 229.67 & 46.34 & 46.14 & 27.90 & 28.45 \\
\hline PROLIN & QANRUT & 0.00 & 134.13 & 135.03 & 32.86 & 32.69 & 18.18 & 18.17 \\
\hline ROPPAN & YUHFEM & 0.00 & 219.68 & 224.17 & 34.55 & 34.47 & 25.21 & 24.45 \\
\hline TAYYOH & TAYYIB & 0.00 & 209.81 & 214.43 & 25.56 & 25.42 & 25.38 & 24.17 \\
\hline TNAPHB & TNAPHC & 0.00 & 279.35 & 281.76 & 32.54 & 32.39 & 30.89 & 30.72 \\
\hline FAFDIA & FAFDOG & 10.41 & 211.50 & 211.57 & 17.86 & 17.92 & 24.69 & 24.68 \\
\hline JAHZIB & TAHFUD & 15.94 & 170.96 & 176.66 & 32.58 & 32.67 & 17.55 & 16.33 \\
\hline NOLCIA & YENDEC & 17.79 & 146.29 & 147.20 & 43 . & 43.89 & 20.71 & 20.56 \\
\hline FARPUK & FASZIJ & 18.09 & 191.17 & 192.60 & 28.65 & 28.76 & 23.37 & 23.17 \\
\hline FOHRIE & MOMKII & 23.13 & 262.05 & 270.09 & 27.76 & 27.90 & 22.66 & 21.23 \\
\hline DIXQIK & KABLOO & 24.95 & 282.69 & 281.32 & 27.86 & 28.01 & 30.26 & 30.65 \\
\hline ZOCPEO & ZOCNUC & 25.60 & 277.48 & 278.35 & 37.89 & 38.05 & 31.21 & 31.10 \\
\hline HUCTIJ & FUJFAS & 27.07 & 261.03 & 264.40 & 28.41 & 28.58 & 34.04 & 33.38 \\
\hline OKOYET & OKOXOC & 29.24 & 232.55 & 231.10 & 33.91 & 34.09 & 26.67 & 27.46 \\
\hline FEPPAR & COQTOR & 34.08 & 223.54 & 223.50 & 23.02 & 23.23 & 22.52 & 22.35 \\
\hline CULGEV & CULGIZ & 38.67 & 228.85 & 221.65 & 23.12 & 23.37 & 23.07 & 23.94 \\
\hline SOGJUU & SOBNED & 48.26 & 164.07 & 166.49 & 24.25 & 24.57 & 19.06 & 18.74 \\
\hline GLUTAM & TACQUJ & 52.99 & 156.29 & 175.93 & 52.18 & 52.53 & 23.39 & 18.34 \\
\hline ZEQVIC & ZERLEP & 53.55 & 100.08 & 99.80 & 30.33 & 30.68 & 14.77 & 14.84 \\
\hline MIJPOK & XENJUX & 55.59 & 203.40 & 197.22 & 23.21 & 23.58 & 17.14 & 18.33 \\
\hline MUKVUJ & MUKVOD & 58.59 & 209.90 & 203.86 & 27.07 & 27.47 & 23.24 & 24.67 \\
\hline XISYAA & HIHRIA & 59.28 & 372.89 & 366.51 & 42.28 & 42.68 & 42.40 & 44.02 \\
\hline OMIVUB & OFIQUR & 61.30 & 207.01 & 205.74 & 31.64 & 32.06 & 25.33 & 25.81 \\
\hline IBUHES & IBUHIW & 61.66 & 250.97 & 248.76 & 28.55 & 28.98 & 25.13 & 25.53 \\
\hline SINYUK & SITFOR & 61.92 & 147.98 & 146.39 & 20.66 & 21.09 & 13.60 & 13.95 \\
\hline APHAMA & APALAM & 62.58 & 281.94 & 278.04 & 38.52 & 38.95 & 31.67 & 32.43 \\
\hline GUWCOQ & HUZCAG & 63.41 & 223.64 & 227.46 & 35.35 & 35.79 & 25.42 & 24.54 \\
\hline ACUFIN & EPTHOX & 63.89 & 245.31 & 244.85 & 28.36 & 28.81 & 28.45 & 28.81 \\
\hline BEKVIY & UTANEJ & 68.05 & 195.42 & 198.29 & 26.63 & 27.12 & 31.06 & 30.28 \\
\hline SUZBIZ & TATHIH & 68.69 & 130.15 & 132.71 & 52.57 & 53.07 & 15.79 & 15.35 \\
\hline
\end{tabular}




\begin{tabular}{|c|c|c|c|c|c|c|c|c|}
\hline Homochiral & Heterochiral & e.e. & $V_{c}$ & $V_{r}$ & $E_{\text {latt }, c}$ & $E_{\text {latt }, r}$ & $E_{d i s p, c}$ & $E_{\text {disp }, r}$ \\
\hline PLACTA & AVIMEY & 70.91 & 201.08 & 192.84 & 28.92 & 29.44 & 22.46 & 24.35 \\
\hline LALNIN & DLALNI & 74.27 & 105.43 & 102.52 & 36.05 & 36.62 & 14.39 & 15.03 \\
\hline EXEWEJ & EXEWAF & 75.19 & 123.58 & 123.77 & 29.61 & 30.19 & 17.01 & 16.85 \\
\hline LVALIN & VALIDL & 76.22 & 149.42 & 143.27 & 37.76 & 38.35 & 18.24 & 19.93 \\
\hline LCYSTN & BOQCUF & 77.34 & 131.43 & 131.70 & 38.53 & 39.14 & 16.53 & 16.49 \\
\hline LTYROS & DLTYRS & 77.55 & 207.83 & 204.39 & 52.24 & 52.85 & 25.56 & 26.35 \\
\hline TICLIC & TICLEY & 78.78 & 270.36 & 267.75 & 32.18 & 32.81 & 30.06 & 30.49 \\
\hline KERZUD & LEHQUL & 80.40 & 236.14 & 231.65 & 31.37 & 32.03 & 28.45 & 29.54 \\
\hline VUGMEQ & VUGMAM & 81.71 & 290.08 & 288.16 & 41.24 & 41.92 & 35.86 & 36.06 \\
\hline RIGZAJ & AXALER & 81.83 & 211.29 & 203.76 & 27.25 & 27.93 & 24.79 & 26.89 \\
\hline PEDZEE & CAHYAN & 85.39 & 239.94 & 240.80 & 25.67 & 26.42 & 26.11 & 26.04 \\
\hline SOXGES & GOMWIP & 87.63 & 203.59 & 190.58 & 64.33 & 65.13 & 20.70 & 23.48 \\
\hline IMITED & IMITIH & 87.66 & 266.56 & 258.46 & 27.78 & 28.58 & 26.36 & 28.57 \\
\hline SIBJIX & ENXBCO & 88.12 & 155.29 & 150.90 & 22.09 & 22.91 & 18.88 & 19.97 \\
\hline EGOPUL & XATQUE & 88.36 & 209.77 & 204.47 & 27.87 & 28.69 & 23.04 & 24.47 \\
\hline KEGYIF & XАTFOP & 88.48 & 276.47 & 269.95 & 29.47 & 30.30 & 26.70 & 28.30 \\
\hline WEGTOR & WEGXEL & 90.39 & 242.96 & 246.30 & 24.49 & 25.37 & 23.91 & 23.20 \\
\hline EWOTUF & JAYNOM & 90.49 & 197.86 & 195.46 & 31.73 & 32.61 & 22.70 & 23.14 \\
\hline NMLALA & RMNALA & 90.50 & 218.48 & 213.02 & 23.95 & 24.84 & 22.92 & 24.25 \\
\hline TOYWAF & WESBEB & 90.78 & 188.21 & 179.38 & 31.06 & 31.95 & 19.72 & 21.57 \\
\hline DEYFOD & CERGEL & 91.39 & 182.87 & 187.96 & 40.44 & & & 21.16 \\
\hline ROPPER & YUHFOW & 92.02 & 226.38 & 225.57 & 35.11 & 36.05 & 26.49 & 26.91 \\
\hline ZZZBLS & KIGCAE & 92.72 & 245.62 & 247.71 & 27.31 & 28.28 & 24.26 & 23.82 \\
\hline CIDTOY & WIKCUO & 93.89 & 168.62 & 168.41 & 23.78 & 24.81 & 18.25 & 18.36 \\
\hline DIQTOO & UGIKOL & 93.92 & 232.69 & 230.75 & 28.93 & 29.95 & 27.35 & 27.76 \\
\hline ANCLEU & IKIDAH & 94.30 & 203.39 & 190.90 & 25.35 & 26.39 & 18.37 & 21.01 \\
\hline WUQLAW & WUQKUP & 95.21 & 234.21 & 228.48 & 33.66 & 34.76 & 26.78 & 27.68 \\
\hline IBUHOC & IBUHUI & 95.33 & 252.43 & 253.00 & 30.88 & 31.98 & 27.00 & 26.76 \\
\hline ICRFRB & ICRFRA & 95.70 & 314.61 & 313.34 & 42.19 & 43.32 & 30.76 & 31.62 \\
\hline ANONEX & KACXAP & 96.01 & 315.50 & 326.87 & 47.88 & 49.04 & 34.67 & 33.35 \\
\hline LEUCIN & DLLEUC & 96.44 & 182.13 & 178.09 & 38.26 & 39.45 & 12.71 & 13.80 \\
\hline LISLEU & DLILEU & 96.77 & 174.18 & 169.72 & 39.64 & 40.86 & 21.30 & 22.71 \\
\hline HESXUZ & IZAMAX & 96.83 & 242.53 & 255.06 & 29.40 & 30.62 & 26.77 & 23.98 \\
\hline QOQHIP & FICWAR & 97.41 & 221.25 & 218.77 & 27.59 & 28.87 & 20.73 & 21.52 \\
\hline LUNQOA & LUNQUG & 97.74 & 268.87 & 265.53 & 31.38 & 32.70 & 28.34 & 28.98 \\
\hline HUQWUL & MOJPOQ & 98.13 & 265.53 & 257.74 & 24.85 & 26.23 & 28.48 & 30.48 \\
\hline JUQCUT & FONNEC & 98.20 & 271.41 & 269.23 & 25.03 & 26.42 & 28.59 & 29.38 \\
\hline LCARVX & CARVOX & 98.20 & 226.56 & 223.63 & 26.40 & 27.79 & 26.19 & 26.88 \\
\hline ALFUCO & ADLFUC & 98.42 & 174.34 & 170.79 & 38.18 & 39.61 & 22.77 & 23.68 \\
\hline OFEQUM & ODETOH & 98.45 & 212.96 & 206.54 & 27.54 & 28.98 & 21.85 & 23.24 \\
\hline YUFCUY & VALNOM & 98.87 & 268.42 & 260.61 & 20.58 & 22.11 & 24.76 & 26.40 \\
\hline AZOZAQ & AZOYUJ & 99.08 & 214.97 & 206.42 & 28.86 & 30.45 & 24.23 & 25.83 \\
\hline FIMZIL & MODBOY & 99.18 & 244.54 & 234.44 & 30.75 & 32.38 & 26.29 & 28.49 \\
\hline SAKRUT & SAKRIH & 99.26 & 356.67 & 372.61 & 50.13 & 51.78 & 39.48 & 36.88 \\
\hline LNLEUC & DLNLUA & 99.32 & 184.41 & 176.37 & 36.71 & 38.39 & 19.13 & 20.61 \\
\hline SAWJIJ & PIMTAZ & 99.38 & 242.44 & 238.52 & 26.67 & 28.38 & 29.94 & 30.84 \\
\hline XAYJAI & XAYHUA & 99.58 & 371.75 & 369.85 & 41.59 & 43.41 & 39.28 & 38.85 \\
\hline AZEHET & SULZUV & 99.90 & 224.53 & 213.00 & 22.64 & 24.89 & 20.61 & 23.23 \\
\hline
\end{tabular}




\begin{tabular}{ll|c|cc|cc|cc}
\hline Homochiral & Heterochiral & e.e. & $V_{c}$ & $V_{r}$ & $E_{\text {latt }, c}$ & $E_{\text {latt }, r}$ & $E_{\text {disp }, c}$ & $E_{\text {disp }, r}$ \\
\hline LHISTD & DLHIST & 99.90 & 175.69 & 166.81 & 47.32 & 49.56 & 21.97 & 24.00 \\
ABINOS & ABINOR & 99.92 & 147.74 & 146.80 & 38.16 & 40.47 & 21.64 & 22.03 \\
RUZYIV & RUZYER & 99.99 & 547.02 & 535.50 & 53.10 & 55.91 & 58.63 & 60.23 \\
LSERIN & DLSERN & 100.00 & 111.26 & 112.20 & 40.25 & 44.15 & 16.23 & 15.95 \\
\hline
\end{tabular}


Figure 1: Non-covalent interaction plots for the homochiral (left) and heterochiral (right) forms of serine (top), glutamine (middle), and glutamic acid (bottom), each with an isovalue of $s=0.6$.
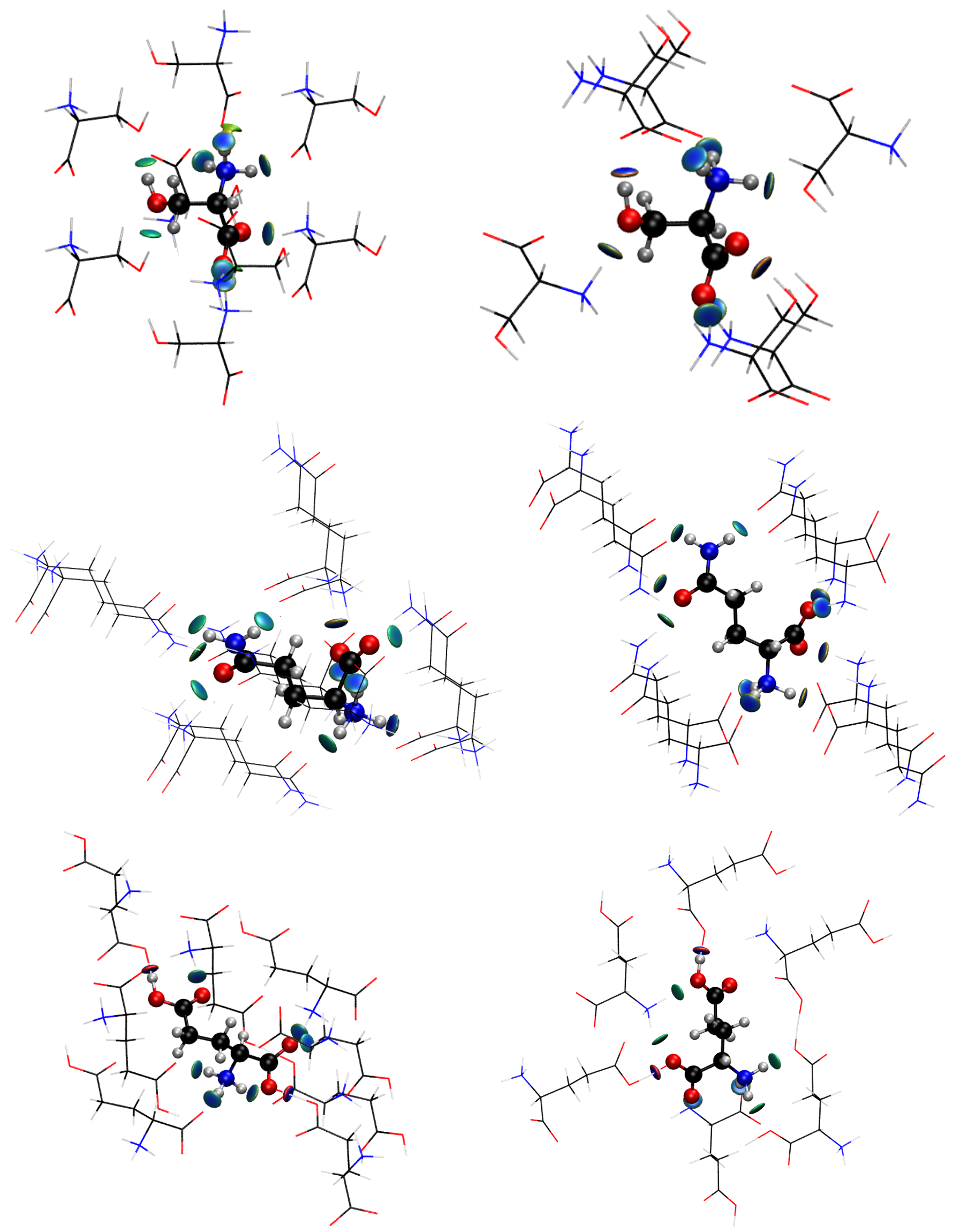